\title{
Level of Organochlorine Pesticide Residues in selected Consumable Vegetables commonly sold in Benin City Markets
}

\section{*ATUANYA, EI; ONUOHA, T}

\author{
Department of Microbiology, University of Benin, Benin City, Nigeria \\ *Corresponding Author Email: onuohakelechi6@ gmail.com
}

\begin{abstract}
This research studied the levels and human health risk assessment of pesticide residues in consumable vegetables such as watermelon, cucumber and onions commonly sold in Benin City markets. A total of twenty-five organochlorine pesticide (OCP) residues in these samples were analyzed using with gas chromatography- electron captured detector (ECD).The estimated acceptable daily intake (EADI) of gamma lindane concentration in fruit vegetable (watermelon, onions and cucumber) was determined based on European union (EU) and United States Agency of Toxic Substances and Disease Registry (ATSDR) standards, using two population age groups (child and adult). The results revealed the levels of organochlorine pesticide residues among the fruit vegetables (watermelon, onions and cucumber). Gamma lindane had the highest value of $0.0002 \mathrm{mg} / \mathrm{kg}$ among the OCP residues detected in fruit vegetable (watermelon). Onions and cucumber were below detection limit $(0.00 \pm 0.00)$. The study, thus suggest that OCPs concentration (gamma lindane) in watermelon is unsafe for human. The study therefore calls for continuous monitoring of agricultural farmlands because continuous exposure to pesticide contaminated food products sold in Benin City markets (watermelon) could affect the health of consumers.
\end{abstract}

\section{DOI: https://dx.doi.org/10.4314/jasem.v22i10.17}

Copyright: Copyright $\odot 2018$ Atuanya and Onuoha. This is an open access article distributed under the Creative Commons Attribution License (CCL), which permits unrestricted use, distribution, and reproduction in any medium, provided the original work is properly cited.

Dates: Received: 17 September 2018; Revised: 24 October: 2018; Accepted: 27 October 2018

Keywords: Pesticide residues, watermelon, cucumber, onions

Pesticides are chemical substances widely used in agriculture to increase the yield, improve the quality, and extend the storage life of food crops(Chapman, 2002). They provide society with a wide range of benefits, especially in agricultural productivity and in the control of diseases. There has been an increasing concern about dietary ingestion of pesticide residues by adults and children who consume fruit and vegetables. Consumption of fruits and vegetables has increased substantially over time because of its health benefit. Xenobiotics are a major cause for concern all over the world, given their persistence to their metabolite residues after degradation by artificial or natural means and adverse effects on humans (Chapman, 2002).In 2001, about 74 percent of agricultural products (fruit, vegetables) in the United States of America (USA) were reported to contain atleast one pesticide residue greater than the acceptable level Adeyemi et al., (2011). This increase in level of pesticide residues has risen in developing countries and the fastest growing markets in Africa, Asia, South and Central America, Eastern Mediterranean Adeyemi et al., (2011). The toxicity of these pesticides has caused them to be banned in developed and many developing nations. Moreover, some developing countries still use such pesticides Adeyemi et al., (2011).

In Nigeria, there have been reports of some levels of pesticide residues in ready to eat agricultural fruit vegetables Papadakis et al., (2015). The results have continually revealed contamination by organochlorine pesticides in agricultural produce sold in Benin City markets (). The organochlorine pesticides (OCPs) include aldrin, endrin, dieldrin, chlordane, heptachlor, DDT, toxaphene, endosulfan, and hexachlorobenzene (HCB) Papadakis et al., (2015). Several activities (inadequate training of personnel, inappropriate use of pesticides, waste from industrial chemical production, and pesticides runoff from agricultural areas, sewage and refuse dump) have contributed to the levels of chlorinated hydrocarbon compounds in agricultural produce sold in Nigeria markets Adeyemi et al., (2011). Because of their potency, efficiency and low cost compared with alternative pesticides, organochlorine pesticides (OCPs) are still being used by some farmers Akinnifesi et al., (2006). The use of OCPs such as DDT has been outlawed since 1990 in Nigeria. These compounds are characterized by high persistent, low polarity, low aqueous solubility and high lipid solubility (lipophilicity). They are ecotoxic, 
non-biodegrable and able to bioaccumulate and biomagnify in humans via consumption of contaminated fruit vegetables which may pose health risk to human Afful et al., (2010). Estimationof potential risk of pesticide exposure to non-target organisms involves the use of the risk quotient (RQ) method. Risk quotient (RQ) is the ratio of the measured environmental concentration (MEC) to the predicted no effect concentration (PNEC). The predicted no effect concentration (PNEC) was obtained by multiplying the $\mathrm{LC}_{50}$ with an assessment factor (AF) of 100. The assessment factor takes into account the uncertainty in extrapolation from laboratory toxicity tests for a limited number of dietary and non-dietary products to the real environment. The $\mathrm{LC}_{50}$ was obtained from (Chapman, 2002) the risk quotient Papadakis et al., (2015).For human health risk estimations, two population groups (young children and adults) the estimated acceptable daily intake (EADI) was used. EADI was obtained by multiplying the residual pesticide concentration $(\mu \mathrm{g} / \mathrm{kg})$ in each ready to eat vegetables by the consumption rate in Nigeria (L/day or $\mathrm{kg} /$ day) and dividing the product by the body weight $(\mathrm{kg}) \mathrm{EADI}=\mathrm{C}$ x CR/BW Fianko et al., (2011).

The aim of this study were to assess the level of organochlorine pesticide residues in ready to eat vegetables (onions, cucumber and watermelon) sold in markets in Benin City.

\section{MATERIALS AND METHOD}

Study area: Benin City have alot of fruit vegetable markets located in different localities in the city where they are sold for final consumption. The markets include Ekiosa, New Benin, Oba market, Ikpoba hill market and Uselu market. The fruit vegetables (Onions, cucumber and watermelon) are usually transported from within the state or other neighboring western, eastern, southern or northern part of the country.

Sample Collection: Random sampling of fruit vegetables; Cucumber, water melon and onions were purchased from the various markets in Benin City. Samples were enclosed in a clean blotting paper and wrapped inside a clean, paper envelope. The addition of a small sachet of silica gel to the envelope helps to reduce the moisture content of the system. However, samples were provided to the analytical laboratory for GC analysis Anastassiades et al., (2003)

Sample Extraction: Prepare a solvent 50:50 mix of Acetone and n-Hexane or DCM and Acetone. Measure about 30 gram aliquot of well mixed sample into a solvent rinsed beaker. Add $1 \mathrm{ml}$ of
Decachlorobiphenyl, mix thoroughly using glass stir rod. Weigh an equal amount of Sodium Sulfate and add to the sample and mix thoroughly to form a free flowing powder. Add 50ml of the solvent mix to the samples. Place sample in the Sonicator and Sonicate for about $10-15$ minutes at about $60^{\circ} \mathrm{C}$. Decant the extract into a round bottom flask. Repeat once more with an additional $50 \mathrm{ml}$ of solvent mix, Sonicate and allow the beaker to settle and decant into the same round bottom flask. Concentrate the sample extract using Rotary Evaporator, to 1-2mLAdd about $5 \mathrm{~mL}$ Hexane to the extract. Evaporate it to reduce the volume to $1-2 \mathrm{~mL}$. Make the final volume $2 \mathrm{~mL}$ in Hexane, giving final sample weight of $15 \mathrm{~g} / \mathrm{mL}$. The sample is ready for Sulfuric acid/Permanganate cleanup/gel permeation chromatography Anastassiades et al., (2003).

Sample Cleanup: Using a syringe or a volumetric pipette, transfer 1 or $2 \mathrm{~mL}$ of the hexane extract into a $10 \mathrm{~mL}$ vial in a fume cupboard. Carefully add $5 \mathrm{~mL}$ of the 1:1 Sulfuric acid: water mixture, ensure there is no exothermic reaction. Cap the cap tightly and vortex for 1-2mins.Allow the phases to separate for at least 3 mins, observe the upper hexane layer and ensure that it is not highly colored. If the upper layer is colored or the emulsion persist, discard the sulfuric acid layer using pipette and add fresh $5 \mathrm{~mL}$ sulfuric acid/water mixture. Cap tightly and vortex as above. Allow the Phases to separate by allowing to stand for 2 to 3 mins.If the upper layer is clear, transfer the Hexane layer using a glass pipette into the $10 \mathrm{~mL}$ vial. Add $1 \mathrm{~mL}$ of $\mathrm{n}$-Hexane to the sulfuric acid layer and repeat the vortex procedure. Allow the phases to separate, transfer the $n-H e x a n e$ layer into the $10 \mathrm{~mL}$ vial. Take care not to include the lacid layer into the $10 \mathrm{~mL}$ vial as it could damage the column and instrument Anastassiades et al., (2003).

Permanganate cleanup: This cleanup procedure is employed when the sulfuric acid cleanup could not remove all the color from the sample extract. Add $5 \mathrm{~mL}$ of $5 \%$ permanganate to the combined $n$-hexane extract above in the hood. Ensure there is no exothermic reaction, cap the vial and vortex for 1 min. Allow the two phases to separate, if a clean separation is achieved, transfer the hexane layer into a clean $10 \mathrm{~mL}$ vial. Add additional $1 \mathrm{~mL}$ hexane to the permanganate layer. Cap the vial and vortex for $1 \mathrm{~min}$. Allow the phase to separate, and transfer the hexane to the $10 \mathrm{ml}$ vial using a glass pipette. Reduce the combined hexane extract volume to the initial $1-2 \mathrm{~mL}$ using the appropriate method. Any remaining Organochlorine Pesticides could be removed using the silica gel cleanup method Anastassiades et al., (2003). 
Silica gel cleanup: Reduce the extract volume to $2 \mathrm{~mL}$ and the extract solvent should be hexane. Mount the clean polypropylene cartridge on a manifold or stand. Block the base with sterile cotton wool or glass wool, whichever is available. Add $2 \mathrm{~g}$ of the activated silica gel to the cartridge and tap the wall of the cartridge for good packing. Add a layer of dry $\mathrm{Na}_{2} \mathrm{SO}_{4}$ to the top of the column. Condition the column with about $15 \mathrm{~mL}$ of n-Hexane, collect the eluate to waste. Do not allow the column to dry up at this stage. Transfer the $2 \mathrm{~mL}$ extract to the head of the column with the help of a pipette. Elute the column with $15-20 \mathrm{~mL}$ hexane, collect the fraction in a clean $50 \mathrm{~mL}$ glass beaker. This fraction contains all the OCPs. Dry the sample extract using the appropriate technique. Make up the dry extract with $2 \mathrm{~mL}$ n-Hexane and transfer into a GC vials. Sample extract is ready for GC analysis Anastassiades et al., (2003).

Calculation for sample analysis: The concentration of each analyte range in a sample was calculated directly from the Instrument using the Data Analysis Software. The final sample weight and the dilution factor can be use in the batch file and the final results would be generated by the software or the use of the Response Factor Method could be employed (Renwick, 2002).

$$
C_{f}=\frac{\text { Area }_{p} \times R_{f} \times V_{f} \times D_{f} \times 100}{W_{i}}
$$

Where $\mathrm{Cf}=$ Final Sample concentration $(\mathrm{mg} / \mathrm{L})$; Area $(\mathrm{p})=$ Measured area of peak (peaks); Wi = Initial weight extracted ( $\mathrm{g}$ dry weight); Vf = Final extract volume $(\mathrm{mL}) ; \mathrm{Df}=$ Dilution factor of sample or extract if diluted and $\mathrm{Rf}=\mathrm{Response}$ factor from the calibration standard calculation. The Df may be calculated as

$$
D_{f}=\frac{\text { Concentration }(P)}{\operatorname{Area}(P)}
$$

Where Concentration $(\mathrm{p})=$ Concentration of peak or total concentration of range and area $=$ Area of peak or total across range.

Risk Assessment of Pesticide Residues: Ecological Risk Assessment: Risk quotient (RQ) method was used to determine risk of pesticide exposure to non-target ready to eat vegetables. RQ is the ratio of the measured environmental concentration (MEC) to the predicted no effect concentration (PNEC). The predicted no effect concentration (PNEC) was obtained by multiplying the $\mathrm{LC}_{50}$ with an assessment factor (AF) of 100. The assessment factor takes into account the uncertainty in extrapolation from laboratory toxicity tests for a limited number of dietary and non-dietary products to the real environment (Chapman, 2002). The $\mathrm{LC}_{50}$ was obtained from (Chapman, 2002), while the RQ Papadakis et al., (2015).may be calculated from

$$
\text { Risk Quotient }(R Q)=\frac{M E C}{P N E C}
$$

Health Risk Estimations: To assess the health risk associated with exposures to pesticide residues through consumption of ready to eat vegetables (dietary).The guidelines for potential risk assessment drawn up by the USFDA were used.

Human Health Risk Assessment of Pesticide Residues in Ready to eat vegetables (Dietary): To estimate the carcinogenic and non-carcinogenic risk of detected pesticides to humans, using two population groups (young children and adults) the estimated acceptable daily intake (EADI) was used. EADI was obtained by multiplying the residual pesticide concentration $(\mu \mathrm{g} / \mathrm{kg})$ in each ready to eat vegetables by the consumption rate in Nigeria (L/day or $\mathrm{kg} /$ day) and dividing the product by the body weight $(\mathrm{kg})$ Fianko et al., (2011). The hazard quotient (HQ) was then obtained from the ratio of EADI and reference dose. The reference dose (RFD) of each pesticide is the exposure that is likely to be without an appreciable risk of deleterious effects and was provided by the USFDA (1999). The food and agricultural organization (FDA, 1999) quotes the per capita consumption of ready to eat vegetables in Nigeria as $9 \mathrm{~kg}$. The following formula was used to estimate the dietary intake. $\mathrm{EADI}=\mathrm{C} \times \mathrm{CR} / \mathrm{BW}$ Fianko et al., (2011).

EADI is the estimated average daily intake, $\mathrm{C}$ is the concentration of pesticide residues, CR represents consumption rate of each ready to eat vegetable while BW represents the body weight of age group. The food and agricultural organization (FDA, 1999) quotes the per capita consumption of ready to eat vegetable in Nigeria as $9 \mathrm{~kg}$., while body weight was set at $70 \mathrm{~kg}$ for adult population group.Hazard quotient (HQ): Hazards quotients were obtained by dividing the EADI by their corresponding reference dose (RfD). Hazard quotient (HQ) =EADI/RfD Fianko et al., (2011).Hazard index (HI): using the hazard quotient derived from Eq. 3, the hazard index (HI) was obtained. Hazard index is used to assess the risk involved in exposure to mixtures of the detected pesticides belonging to the same chemical group (organochlorines).Hazard Index (Fiankoet al., 2011).

$$
(\mathrm{HI})=\sum_{i}^{n} H Q l .
$$




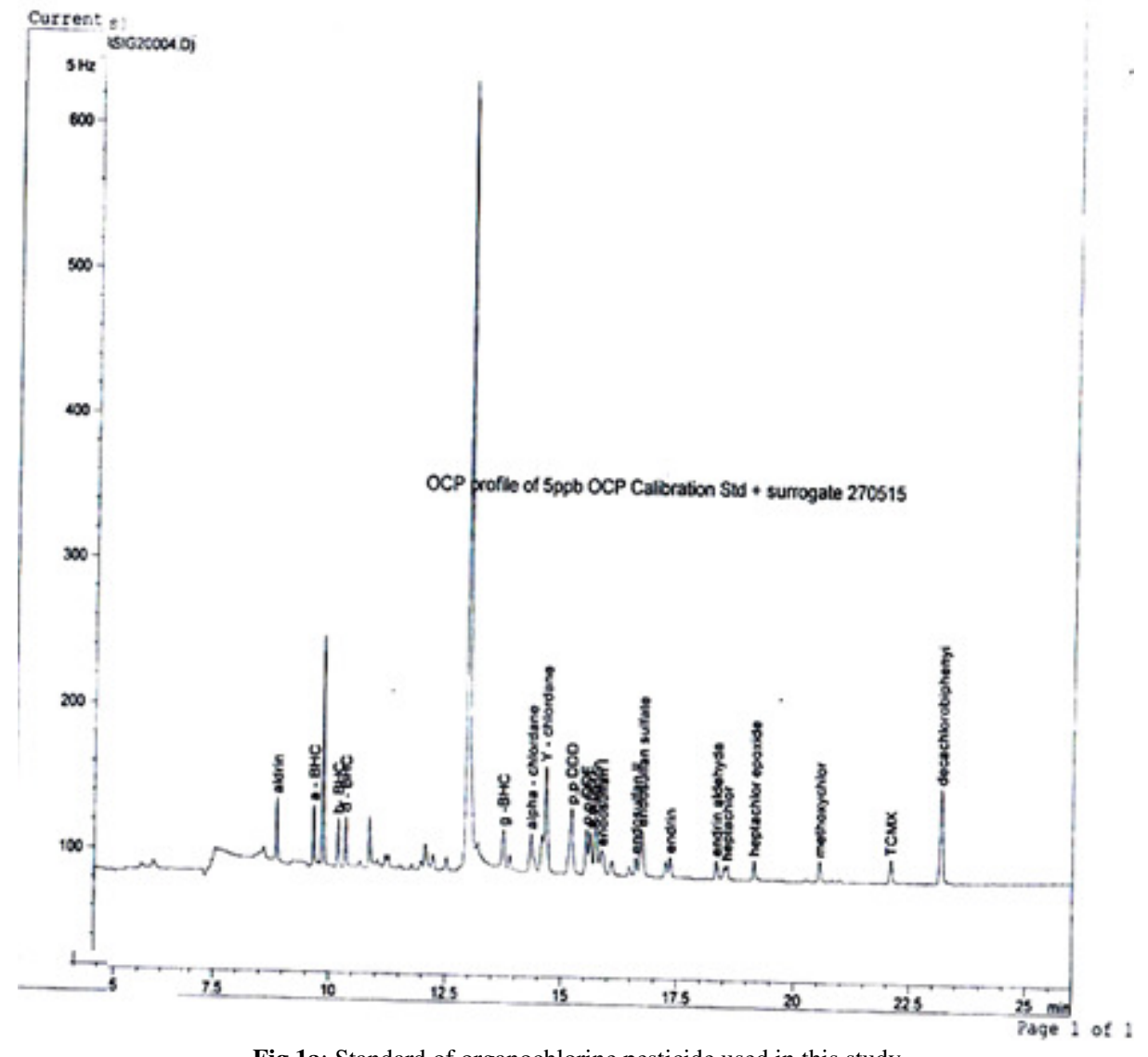

Fig 1a; Standard of organochlorine pesticide used in this study

\section{RESULTS AND DISCUSSION}

The concentration of pesticide residues in fruit vegetables (watermelon, onions and cucumber) sold in Benin City, Edo state has been demonstrated. Figure $1 \mathrm{a}, \mathrm{b}, \mathrm{c}$ and d shows the representative chromatogram standard and samples. No interference peaks were obtained for the blank sample chromatogram at the same retention time as the targets compounds. The mean recovery values for the spiked samples are shown in Table 1. The procedure employed in this study is reproducible, efficient and reliable for the analysis of OCPs as stipulated by EU guidelines for evaluating accuracy and precision method (European Union, 2005). The chromatogram result in this study is in consonance with that of Papadakis et al., (2015) who assessed fruit vegetables in Addo Ekitti, Ogun and Ghana. Reports from this investigation reveal a great deal of organochlorine pesticide residues ranged from BL (Below detection limit) to $0.0002 \mathrm{mg} / \mathrm{kg}$ (Table1). The presence of these organochlorine pesticide residues in fruit vegetable (watermelon) showed that farmers still use them for the control of pest. This is because of their potency, efficiency and low cost compared with alternative pesticides (banned or not) Akinnifesi et al., (2006).

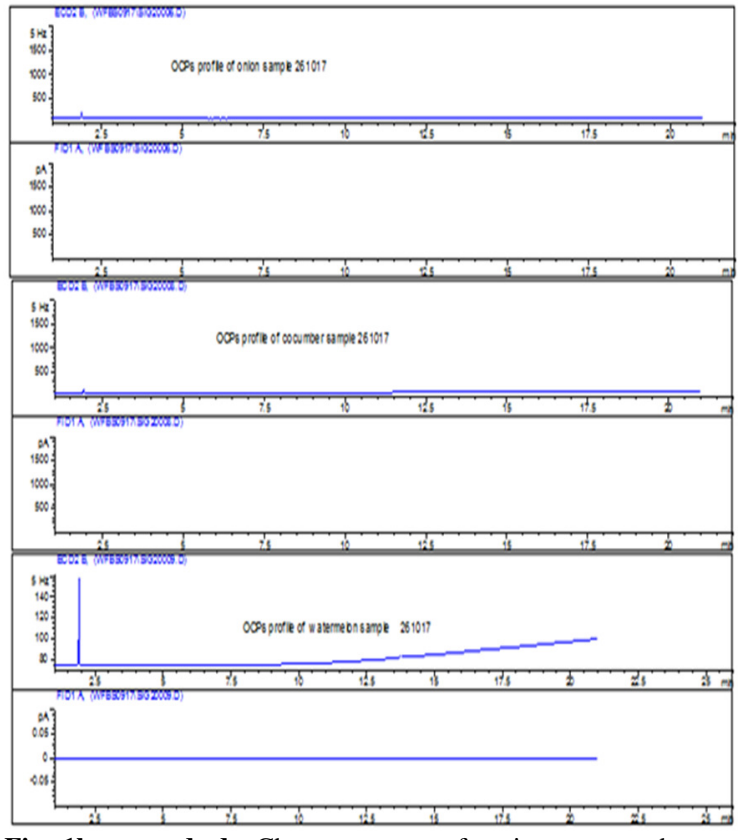

Fig 1b, c and d; Chromatogram of onions, cucumber and watermelon samples analysedTwenty five (25) pesticides were assessed in these consumable products (Table.1) with gamma lindane having the highest mean concentration $(0.0002 \mathrm{mg} / \mathrm{kg})$ in watermelon sample. 
Several activities (volatilization, photolysis, penetration through the plant surface, inadequate training of personnel, and inappropriate use of pesticides) could be attributed to the levels of chlorinated hydrocarbon compounds in agricultural produce sold in Benin City markets (Adeyemi et al., 2011). The findings in this study is in consonance with the findings of (FDA, 1999) observed that organochlorine pesticide residue in watermelon ranged from 0.0001 to $0.75 \mathrm{mg} / \mathrm{kg}$ in Benin City, Addo Ekitti, Ogun, Lagos and Ghana metropolis. Previous studies have demonstrated that organochlorine pesticide like gamma lindane is toxic and can affect non target organisms other than the organisms of interest, thereby causing great menace to ecosystem and to consumers Papadakis et al., (2015). The use of organochlorine pesticides for the control of pest by farmers is a global issue. These compounds are characterized by high persistent, low polarity, low aqueous solubility and high lipid solubility (lipophilicity). They are ecotoxic, non-biodegrable and able to bioaccumulate and biomagnify in living organisms Afful et al., (2010). The major concerns are their toxic effects such as interfering with the reproductive systems and foetal development as well as their capacity to cause cancer, cardiovascular disease, asthma and other health related diseases. The ecological and human health risk assessment of dietary intake of organochlorine pesticide concentration (gamma lindane) in watermelon in this study is shown in (Table 2). The risk quotient, estimated acceptable daily intake (EADI), hazard quotient (HQ) and hazard index (HI) for gamma lindane in watermelon sample were calculated using two population groups (child and adult) with varying body weights (35 and $70 \mathrm{~kg}$ ) (Chapman, 2002). The study revealed that the risk quotient (RQ) of gamma lindane were (5.0E-07), estimated acceptable daily intake for both population groups were (EADI) (1.8E-04) and (2.57E-05).

Table 1; Mean concentration of pesticide residues in ready to eat vegetables

\begin{tabular}{llll}
\hline COMPONENT & Onions & Cucumber & Watermelon \\
\hline ALDRIN & $0.00 \pm 0.00$ & $0.00 \pm 0.00$ & $0.00 \pm 0.00$ \\
a - BHC & $0.00 \pm 0.00$ & $0.00 \pm 0.00$ & $0.00 \pm 0.00$ \\
b - BHC & $0.00 \pm 0.00$ & $0.00 \pm 0.00$ & $0.00 \pm 0.00$ \\
d - BHC & $0.00 \pm 0.00$ & $0.00 \pm 0.00$ & $0.00 \pm 0.00$ \\
Gamma - BHC (LINDANE) & $0.00 \pm 0.00$ & $0.00 \pm 0.00$ & $0.0002 \pm 0.00$ \\
ALPHA - CHLORDANE & $0.00 \pm 0.00$ & $0.00 \pm 0.00$ & $0.00 \pm 0.00$ \\
GAMMA - CHLORDANE & $0.00 \pm 0.00$ & $0.00 \pm 0.00$ & $0.00 \pm 0.00$ \\
ATRAZINE & $0.00 \pm 0.00$ & $0.00 \pm 0.00$ & $0.00 \pm 0.00$ \\
p,p DDD & $0.00 \pm 0.00$ & $0.00 \pm 0.00$ & $0.00 \pm 0.00$ \\
p,p DDE & $0.00 \pm 0.00$ & $0.00 \pm 0.00$ & $0.00 \pm 0.00$ \\
p,p DDT 4, 4 DDT & $0.00 \pm 0.00$ & $0.00 \pm 0.00$ & $0.00 \pm 0.00$ \\
DIEDRIN & $0.00 \pm 0.00$ & $0.00 \pm 0.00$ & $0.00 \pm 0.00$ \\
ENDOSULFAN 1 & $0.00 \pm 0.00$ & $0.00 \pm 0.00$ & $0.00 \pm 0.00$ \\
ENDOSULFAN 11 & $0.00 \pm 0.00$ & $0.00 \pm 0.00$ & $0.00 \pm 0.00$ \\
ENDOSULFAN SULFATE & $0.00 \pm 0.00$ & $0.00 \pm 0.00$ & $0.00 \pm 0.00$ \\
ENDRIN & $0.00 \pm 0.00$ & $0.00 \pm 0.00$ & $0.00 \pm 0.00$ \\
ENDRIN ALDEHYDE & $0.00 \pm 0.00$ & $0.00 \pm 0.00$ & $0.00 \pm 0.00$ \\
HEPTACLOR & $0.00 \pm 0.00$ & $0.00 \pm 0.00$ & $0.00 \pm 0.00$ \\
HEPTACHOR EPOXIDE & $0.00 \pm 0.00$ & $0.00 \pm 0.00$ & $0.00 \pm 0.00$ \\
METHOXYCHLOR & $0.00 \pm 0.00$ & $0.00 \pm 0.00$ & $0.00 \pm 0.00$ \\
DIAZINON & $0.00 \pm 0.00$ & $0.00 \pm 0.00$ & $0.00 \pm 0.00$ \\
PHOSPHORO METHYL GLYCINE & $0.00 \pm 0.00$ & $0.00 \pm 0.00$ & $0.00 \pm 0.00$ \\
TCMX & $0.00 \pm 0.00$ & $0.00 \pm 0.00$ & $0.00 \pm 0.00$ \\
CARBAMATE & $0.00 \pm 0.00$ & $0.00 \pm 0.00$ & $0.00 \pm 0.00$ \\
DECACHLOROBIPHENYL & $0.00 \pm 0.00$ & $0.00 \pm 0.00$ & $0.00 \pm 0.00$ \\
TOTAL OCP (mg/Kg) & $\mathbf{B D L}$ & $\mathbf{B D L}$ & $\mathbf{0 . 0 0 0 2} \pm 0.00$ \\
\hline
\end{tabular}
Key: OCP; Organochlorine pesticide residue, BDL;Below detection limit

Table 2: Estimated accetable daily intake (EADI) and hazard quotient (HQ) for OCPs in consumption of watermelon

\begin{tabular}{llllllll}
\hline 'Pesticide & Concentration & CR & child & & Adult & RQ \\
\hline Gamma- BHC & 0.0002 & 9 & EADI & HQ & EADI & HQ & \\
\cline { 5 - 6 } (Lindane) & & & $1.8 \mathrm{E}-04$ & 0.6 & $2.57 \mathrm{E}-05$ & 0.08 & \\
Hazard index & & 0.6 & & 0.08 & & $5.0 \mathrm{E}-07$ \\
\hline
\end{tabular}

The hazard quotient (HQ) estimated were (0.6) and (0.08). The hazard index (HI) were (5.0E-07), (0.6 and 0.08). Aldrin, a-BHC, b-BHC, Alpha-chlordane, Gamma chlordane, Atrazine, P'p DDD, P'P DDE, P'p
DDT 4, 4DDT, Diedrin, Endosulfan 1, Endosulfan 11, Endosulfan sulfate, Endrin, Endrin aldehyde, Heptachlor, Heptachlor epoxide, Methoxychlor, Diazinon, Phosphoromethyl glycine, TCMX, 
Carbamate and Decachlorobiphenyl were below detection limit (BL). In another recent study carried out by Papadakis et al., (2015) revealed similar results in Ado Ekiti $(0.000$ to $0.0005 \mathrm{mg} / \mathrm{kg})$ of gamma lindane in fruit vegetables estimated daily. Organochlorine pesticide residues at any given concentration are highly toxic, bioaccumulate and not readily biodegraded (FDA, 1999). Though the estimated concentrations in this study were minute, ecological risk assessment showed that there is a potential of toxic effects to fruit vegetable (watermelon) upon exposure to organochlorine pesticides. Risk projections for humans from dietary intake also revealed that there is potential for cancer effects. Projections showed that both children and adult were at high health risk. The result in this study is in line with the United States Agency of Toxic Substances and Disease Registry (ATSDR, 2002) and European Union (EU, 2005) standard for estimation of OCPs $(0.00001$ to $1 \mathrm{mg} / \mathrm{kg})$ in watermelon that could be considered as unsafe to human.

Conclusion: The study therefore calls for continuous monitoring of agricultural farmlands because continuous exposure to pesticide contaminated food products sold in Benin City markets (watermelon) could affect health of consumers.

\section{REFERENCES}

Adeyemi, D., Anyakora, C., Ukpo, G., Adedayo, A., Darko, G (2011). Evaluation of the levels oforganochlorine pesticide residues in water samples of Lagos Lagoon using solid phase extraction method. J. of Environ. Chem. and Ecotoxicol. 3 (6): 160-166.

Afful, S.,Arim, AK, Sertor-Armah, Y (2010). Spectrum of organochlorine pesticide residues in fish samples from dense basin. Res. J. Environ. Sci. 2 (3): 133-138.
Akinnifesi, TA;Asubiojo, OI,Amusan, AA (2006). Effects of fungicide residues on the physicochemical characteristics of soils of a major cocoa-producing area of Nigeria. Sci. andTotal Environ.366 (2-3): 876-879.

Anastassiades, M., Lehotay, SJ, Štajnbaher, D., Schenck, FJ (2003). Fast and easy multi residue method employing acetonitrile extraction/partitioning and "dispersive solidphase extraction" for the determination of pesticide residues in produce. J. Assoc. Analyt. Comm. Inter. 86: 412-431.

(ATSDR) Agency of Toxic Substances and Disease Registry (2002). Toxicological profile for aldrin and dieldrin. Atlanta. GA, US department of Health and Human Services Public Health Service (p. 116).

Chapman, PM (2002). Integrating toxicology and ecology: putting the "eco" into ecotoxicology. Mar. Polluti. Bull. 44: 7-15.

Fianko, J., Donkor, A., Lowor, S., Yeboah, P., Glover, E., Adom, T., Faanu, A (2011). Health Risk Associated with Pesticide Contamination of Fish and Fruits Vegetables from the Densu River Basin in Ghana. J. Environ. Protect. 3: 125-154.

Papadakis, E; Zisisvryzas, AK; Katerina, K., Konstantinos, M., Papadopoulou-Mourkidou, E (2015). A pesticide monitoring survey in rivers and lakes of northern Greece and its human and ecotoxicological risk assessment. Ecotoxicol. Environ. Safety. 124: 1-15.

Renwick, AG (2002). Pesticide residue analysis and its relationship to hazard characterization (ADI/ARfD) and intake estimations (NEDI/NESTI). Pest.Manage. Sci. 